\title{
The Fuzzy Economic Order Quantity Problem with a Finite Production Rate and Backorders
}

\author{
Kaj-Mikael Björk ${ }^{1,2}$ \\ ${ }^{1}$ Åbo Akademi University, IAMSR, 20520 Turku, Finland \\ ${ }^{2}$ Department of Business, Information Technology and Media, Arcada University of Applied Sciences, \\ 00550 Helsinki, Finland \\ Correspondence should be addressed to Kaj-Mikael Björk, kbjork@abo.fi
}

Received 10 October 2011; Accepted 3 January 2012

Academic Editor: Farid Melgani

Copyright (๑) 2012 Kaj-Mikael Björk. This is an open access article distributed under the Creative Commons Attribution License, which permits unrestricted use, distribution, and reproduction in any medium, provided the original work is properly cited.

The track of developing Economic Order Quantity (EOQ) models with uncertainties described as fuzzy numbers has been very lucrative. In this paper, a fuzzy Economic Production Quantity (EPQ) model is developed to address a specific problem in a theoretical setting. Not only is the production time finite, but also backorders are allowed. The uncertainties, in the industrial context, come from the fact that the production availability is uncertain as well as the demand. These uncertainties will be handled with fuzzy numbers and the analytical solution to the optimization problem will be obtained. A theoretical example from the process industry is also given to illustrate the new model.

\section{Introduction}

The earliest models of batch-production were derived from the basic Economic Order Quantity (EOQ) model in the early 20th century. During this time, mathematical methods started emerging to optimize the size of the inventory and the orders [1], and since then, there have been an increasing number of contributions that complement the basic model in different ways. One of them is the extension of finite production rate and another one is when backordering is allowed. The EOQ models are most often used in a continuous-review setting and it is assumed that the inventory can be monitored every moment in time.

Decision making under uncertainty is nothing new. In Liberatore [2], an EOQ model with backorders is derived through probabilistic means. However, the uncertainties in many supply chains today are inherent fuzzy [3]. This comes from the fact that there are seldom statistical data to support the calculations, but the uncertainty distributions have to be based on expert opinions only. This is typically the case for new products, and other products with very large seasonal variations, for example. In these cases it is often possible to use fuzzy numbers instead of probabilistic approaches
$[4,5]$. There are many research contributions in this line of research. For instance, Ouyang et al. [6,7] allowed the lead times to be decision variables. Salameh and Jaber [8] introduced a model that captured also the defective rate of the goods. Chang [9] worked out some fuzzy modifications of this model. A good review of this research track is found in [10]. Another set of results in this line of research is found in Jaber et al. [11], and Khan et al. [12], where the learning aspect of the inspection of quality was taken explicitly into consideration and Khan et al. [13], where the inspection errors (as well as the imperfect items) also were modeled. There is also a track of solving EOQ models numerically, for instance, in Chang et al. [14] an EOQ with fuzzy backorder quantities. However, analytically solutions are desirable if they are possible to find. Björk and Carlsson [15] and Björk [16] solved the same model (as Chang et al. [17]) analytically. In Björk [18], a fuzzy EPQ model with multi-item, shared production capacity, was introduced and solved analytically. Other fuzzy EOQ models are, for instance, as in Yao et al. [19] that presented a model for two replaceable merchandizes. In order to find analytical solutions for fuzzy EOQ problems, there is usually a need of defuzzifying the model before the optimization procedure. Yao and Chiang [20] used the 
signed distance method to defuzzify an EOQ model without backorders. This was found to be a good way of handling the fuzziness in the models.

The business domain that works as a source of inspiration is found within the Nordic forest industries. For instance, the paper producing companies are often exposed to uncertainties in several dimensions. The decisions made in these supply chains are done under uncertainties that often cannot be captured by probabilistic measures, (cf. [14], [15]). Within the business context, the demand is often uncertain as well as the setup times. There are typically only a few production lines producing a large number of products. This leads to a significant number of setups of the production equipment. The setup times may vary quite much due to different operating behavior (the plants are operated 24/7) and sometimes because of the availability of raw material. These uncertainties can all add up to uncertain inventory levels (and backorder levels). Earlier work that is closely related to the work presented in this article is for instance, the model by Björk [21], which did not allow for backorders and the uncertainties were found only in the total cycle time. Björk [16] allowed for backorders but did not have a finite production rate (i.e., the model was more tuned towards the distributors in the paper supply chain). Björk [18] did the same setup, but the demand was assumed crisp and the triangular fuzzy numbers were assumed to be symmetrical. Therefore there is a need of a model, where the setup times (or in fact the backorder level) and the demand is assumed to be asymmetrical triangular fuzzy numbers. The corresponding crisp model is part of the basic EOQ literature and can be found in Cárdenas-Barrón [22-24], for instance. The work in this paper also extends the excellent work by Kazemi et al. [25], in the sense that they did not consider a finite production time. They did, however, perform a very comprehensive study of a fuzzy EOQ model with backorders.

The analytical solution is desired and therefore the fuzzy model needs to be defuzzified. The convexity of the defuzzified objective function needs to be established (as in Björk [16, 18]). This can be done with the second-order derivatives (i.e., the Hessian), for instance. The paper is organized as follows: the crisp model found in the EOQ literature is presented. Then the fuzzy model relevant to the Nordic process industry is presented and defuzzified with the signed distance method, and the analytical solution is worked out with the first-order derivatives, while the objective function is proven to be strictly convex. Finally an example is given as well as a brief discussion and further research tracks.

\section{The Crisp EOQ Model with Backorders and a Finite Production Rate}

The classical EPQ problem (with backorders) formulation consists of two decision variables, the size of the production batch and the amount of the allowed backorders. The latter variable can be exchanged to the maximum amount of inventory there can be, that is, $I_{\max }$ (cf. Figure 1). Under no

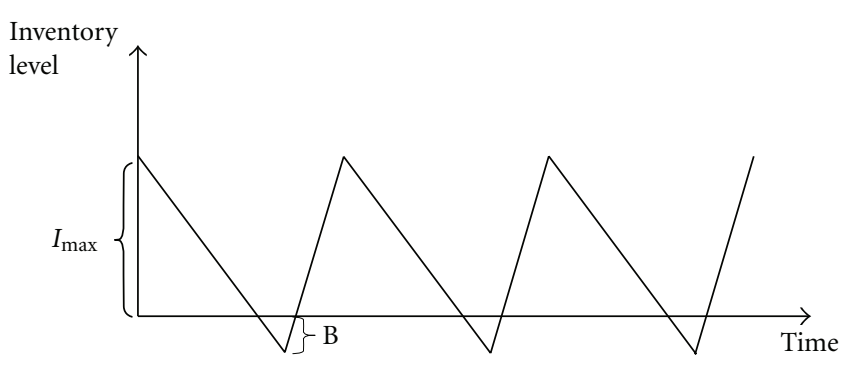

FIgURE 1: The representation of the EPQ model with backorders.

uncertainty, the inventory will have strict seesaw behaviour (cf. Figure 1).

The parameters and variables in the classical EOQ model are the following (the notations are the same as found in Cárdenas-Barrón, [22] to make it easier to read this paper):

$Q$ is the production batch size (variable),

$K$ is the fixed cost per production run (parameter),

$D$ is the annual demand of the product (parameter),

$B$ is maximum shortage (just after a production run starts, variable),

$P$ is the annual production rate (parameter),

$h$ is the unit holding cost per year (parameter),

$b$ is the unit shortage penalty cost per year (parameter),

$I_{\max }$ is the maximum inventory level (just after a production run ends, variable),

$C$ is the total average annual costs for the system (objective value).

The total cost function $C$ is given by (as a basic result in the EOQ-theory)

$$
C(Q, B)=\frac{K D}{Q}+\frac{B^{2} b}{2 Q \rho}+\frac{(Q \rho-B)^{2} h}{2 Q \rho}
$$

In addition, the well-known EOQ-theory will give the following relationship:

$$
I_{\mathrm{Max}}=Q \rho-B
$$

where

$$
\rho=1-\frac{D}{P}
$$


All parameters and variables can be assumed to be strictly greater than 0 . In classical theory the maximal inventory level is given by

$$
Q^{*}=\sqrt{\frac{2 K D}{h \rho}} \cdot \sqrt{\frac{h+b}{b}} .
$$

Classical results give us that the optimal backorder quantity is

$$
B^{*}=\sqrt{\frac{2 K D h \rho}{b(h+b)}} .
$$

\section{The EPQ Model with Backorders and Fuzzy Setup Times and Demand}

Now for the fuzzy uncertainties, we can assume that the setup time is uncertain but possible to describe with a triangular fuzzy number (possibly asymmetric). The setup times are not explicitly in (1). However fuzzy setup times will affect the maximum backorder quantity $B$. The ordering quantity, $Q$, is assumed to be a crisp number since the production wants to have an exact number to produce. The reorder point will also be a fixed inventory level, not affected by the uncertainties.

The setup time is a triangular fuzzy number given by

$$
\widetilde{L}_{e}=\left(L_{e}-\Delta_{l}^{L}, L_{e}, L_{e}+\Delta_{h}^{L}\right)
$$

The maximum backorder level $B$ is

$$
\widetilde{B}=\left(B-\Delta_{l}^{B}, B, B+\Delta_{h}^{B}\right)
$$

where

$$
\Delta_{l}^{B}=\Delta_{h}^{L} \cdot D, \quad \Delta_{h}^{B}=\Delta_{l}^{L} \cdot D
$$

Since annual demand also is uncertain, but it is assumed to be captured by a (possibly asymmetric) fuzzy number:

$$
\widetilde{D}=\left(D-\Delta_{l}^{D}, D, D+\Delta_{h}^{D}\right)
$$

The total annual cost in the fuzzy sense will be

$$
\widetilde{C}(Q, \widetilde{B})=\frac{K \tilde{D}}{Q}+\frac{\widetilde{B}^{2} b}{2 Q \rho}+\frac{(Q \rho-\widetilde{B})^{2} h}{2 Q \rho} .
$$

The strategy in this paper is to first defuzzify the objective function, (10), and then derive the solution analytically. If the signed distance method is used as the defuzzification method, the objective function will be

$$
\begin{aligned}
d(\widetilde{C}, \widetilde{0})= & \frac{K \cdot d(\widetilde{D}, 0)}{Q}+\frac{d\left(\widetilde{B}^{2}, \tilde{0}\right) \cdot b}{2 Q \rho} \\
& +\frac{d\left((Q \rho-\widetilde{B})^{2}, \widetilde{0}\right) h}{2 Q \rho},
\end{aligned}
$$

where, according to (A.4)

$$
\begin{aligned}
d(\tilde{D}, \tilde{0}) & =\frac{1}{4}\left[\left(D-\Delta_{l}^{D}\right)+2 D+\left(D+\Delta_{h}^{D}\right)\right] \\
& =D+\frac{1}{4} \Delta_{h}^{D}-\frac{1}{4} \Delta_{l}^{D}
\end{aligned}
$$

and according to (A.4),

$$
\begin{aligned}
& d\left(\widetilde{B}^{2}, \tilde{0}\right) \\
& =\frac{1}{2} \int_{0}^{1}\left[\left(B^{2}\right)_{L}(\alpha)+\left(B^{2}\right)_{U}(\alpha)\right] d \alpha \\
& =\frac{1}{2} \int_{0}^{1}\left[\left(B-\Delta_{l}^{B}+\Delta_{l}^{B} \alpha\right)^{2}+\left(B+\Delta_{h}^{B}-\Delta_{h}^{B} \alpha\right)^{2}\right] d \alpha \\
& =\frac{1}{2} \int_{0}^{1}\left[B^{2}-B \Delta_{l}^{B}+B \Delta_{l}^{B} \alpha-B \Delta_{l}^{B}+\Delta_{l}^{B^{2}}-\Delta_{l}^{B} \alpha+B \Delta_{l}^{B} \alpha\right. \\
& \quad-\Delta_{l}^{B^{2}} \alpha+\Delta_{l}^{B^{2}} \alpha^{2}+B^{2}+B \Delta_{h}^{B}-B \Delta_{h}^{B} \alpha+B \Delta_{h}^{B}+\Delta_{h}^{B^{2}} \\
& \left.\quad-\Delta_{h}^{B^{2}} \alpha-B \Delta_{h}^{B} \alpha+\Delta_{h}^{B} \alpha+\Delta_{h}^{B 2} \alpha^{2}\right] d \alpha,
\end{aligned}
$$

which finally can be rewritten as

$$
d\left(\widetilde{B}^{2}, \tilde{0}\right)=B^{2}-\frac{1}{2} B \Delta_{l}^{B}+\frac{1}{2} B \Delta_{h}^{B}+\frac{1}{6} \Delta_{l}^{B^{2}}+\frac{1}{6} \Delta_{h}^{B^{2}} .
$$

The third expression to be defuzzified is

$$
\begin{aligned}
& d\left((Q \rho-\tilde{B})^{2}, \tilde{0}\right) \\
& =\frac{1}{2} \int_{0}^{1}\left[(Q \rho-B)_{L}^{2}(\alpha)+(Q \rho-B)_{U}^{2}(\alpha)\right] d \alpha \\
& =\frac{1}{2} \int_{0}^{1}\left[\left(Q \rho-B+\Delta_{l}^{B}-\Delta_{l}^{B} \alpha\right)^{2}+\left(Q \rho-B-\Delta_{h}^{B}+\Delta_{h}^{B} \alpha\right)^{2}\right] d \alpha \\
& =\rho^{2} Q^{2}-2 \rho Q B+B^{2}-\frac{1}{2} B \Delta_{l}^{B}+\frac{1}{2} B \Delta_{h}^{B}+\frac{1}{2} \rho Q \Delta_{l}^{B}-\frac{1}{2} \rho Q \Delta_{h}^{B} \\
& \quad+\frac{1}{6} \Delta_{l}^{B^{2}}+\frac{1}{6} \Delta_{h}^{B^{2}} .
\end{aligned}
$$


In (15) we need to add a very unrestrictive condition that $Q \rho-B-\Delta_{h}^{B}>0$. If the expressions for the defuzzified terms are inserted into (11), we obtain

$$
\begin{aligned}
C(Q, B) \equiv & d(\tilde{C}, \tilde{0}) \\
= & \frac{K D}{Q}+\frac{K \Delta_{h}^{D}}{4 Q}-\frac{K \Delta_{l}^{D}}{4 Q}+\frac{B^{2} b}{2 Q \rho}+\frac{\Delta_{l}^{B^{2}} b}{12 Q \rho}+\frac{\Delta_{h}^{B^{2}} b}{12 Q \rho}+ \\
& \frac{Q \rho h}{2}-h B+\frac{B^{2} h}{2 Q \rho}+\frac{\Delta_{l}^{B^{2}} h}{12 Q \rho}+\frac{\Delta_{h}^{B^{2} h}}{12 Q \rho}+\frac{B \Delta_{h}^{B} b}{4 Q \rho} \\
& -\frac{B \Delta_{l}^{B} b}{4 Q \rho}+\frac{B \Delta_{h}^{B} h}{4 Q \rho}-\frac{B \Delta_{l}^{B} h}{4 Q \rho}+\frac{\Delta_{l}^{B} h}{4}-\frac{\Delta_{h}^{B} h}{4} .
\end{aligned}
$$

For the computation of the Hessian matrix, the derivatives need first to be computed (first and second grades):

$$
\begin{aligned}
\frac{\partial C}{\partial Q}= & -\frac{K D}{Q^{2}}-\frac{K \Delta_{h}^{D}}{4 Q^{2}}+\frac{K \Delta_{l}^{D}}{4 Q^{2}}-\frac{B^{2} b}{2 \rho Q^{2}}-\frac{\Delta_{l}^{B} b}{12 \rho Q^{2}}-\frac{\Delta_{h}^{B^{2}} b}{12 \rho Q^{2}} \\
& +\frac{\rho h}{2}-\frac{B^{2} h}{2 \rho Q^{2}}-\frac{\Delta_{l}^{B^{2}} h}{12 \rho Q^{2}}-\frac{\Delta_{h}^{B^{2}} h}{12 \rho Q^{2}}-\frac{B \Delta_{h}^{B} b}{4 \rho Q^{2}}+\frac{B \Delta_{l}^{B} b}{4 \rho Q^{2}} \\
& -\frac{B \Delta_{h}^{B} h}{4 \rho Q^{2}}+\frac{B \Delta_{l}^{B} h}{4 \rho Q^{2}},
\end{aligned}
$$

$$
H=\left[\begin{array}{c}
\frac{h}{\rho Q}+\frac{b}{\rho Q} \\
-\frac{B b}{\rho Q^{2}}-\frac{B h}{\rho Q^{2}}-\frac{\Delta_{h}^{B} b}{4 \rho Q^{2}}+\frac{\Delta_{l}^{B} b}{4 \rho Q^{2}}-\frac{\Delta_{h}^{B} h}{4 \rho Q^{2}}+\frac{\Delta_{l}^{B} h}{4 \rho Q^{2}}
\end{array}\right.
$$

The first determinant is $(\operatorname{Det} 1=(h / Q \rho)+(b / Q \rho)>0)$, which is necessary for the convexity requirement. The second determinant is given by

$$
\begin{aligned}
\operatorname{Det} 2=\frac{h+b}{Q \rho}\left(\frac{2 K D}{Q^{3}}+\frac{K \Delta_{h}^{D}}{2 Q^{3}}-\frac{K \Delta_{l}^{D}}{2 Q^{3}}+\frac{B^{2} b}{\rho Q^{3}}+\frac{\Delta_{l}^{B} b}{6 \rho Q^{3}}\right. \\
+ \\
\quad \frac{\Delta_{h}^{B^{2}} b}{6 \rho Q^{3}}+\frac{B^{2} h}{\rho Q^{3}}+\frac{\Delta_{l}^{B^{2} h}}{6 \rho Q^{3}}+\frac{\Delta_{h}^{B^{2}} h}{6 \rho Q^{3}}+\frac{B \Delta_{h}^{B} h}{2 \rho Q^{3}} \\
\left.-\frac{B \Delta_{l}^{B} h}{2 \rho Q^{3}}+\frac{B \Delta_{h}^{B} b}{2 \rho Q^{3}}-\frac{B \Delta_{l}^{B} b}{2 \rho Q^{3}}\right) \\
-\left(-\frac{B b}{\rho Q^{2}}-\frac{B h}{\rho Q^{2}}-\frac{\Delta_{h}^{B} b}{4 \rho Q^{2}}+\frac{\Delta_{l}^{B} b}{4 \rho Q^{2}}-\frac{\Delta_{h}^{B} h}{4 \rho Q^{2}}+\frac{\Delta_{l}^{B} h}{4 \rho Q^{2}}\right)^{2}
\end{aligned}
$$

$$
\begin{aligned}
\frac{\partial C}{\partial B}= & \frac{B b}{\rho Q}-h+\frac{B h}{\rho Q}+\frac{\Delta_{h}^{B} b}{4 \rho Q}-\frac{\Delta_{l}^{B} b}{4 \rho Q}+\frac{\Delta_{h}^{B} h}{4 \rho Q}-\frac{\Delta_{l}^{B} h}{4 \rho Q} \\
\frac{\partial^{2} C}{\partial Q^{2}}= & \frac{2 K D}{Q^{3}}+\frac{K \Delta_{h}^{D}}{2 Q^{3}}-\frac{K \Delta_{l}^{D}}{2 Q^{3}}+\frac{B^{2} b}{\rho Q^{3}}+\frac{\Delta_{l}^{B^{2}} b}{6 \rho Q^{3}}+\frac{\Delta_{h}^{B^{2}} b}{6 \rho Q^{3}} \\
& +\frac{B^{2} h}{\rho Q^{3}}+\frac{\Delta_{l}^{B^{2}} h}{6 \rho Q^{3}}+\frac{\Delta_{h}^{B^{2}} h}{6 \rho Q^{3}}+\frac{B \Delta_{h}^{B} b}{2 \rho Q^{3}}-\frac{B \Delta_{l}^{B} b}{2 \rho Q^{3}}+\frac{B \Delta_{h}^{B} h}{2 \rho Q^{3}} \\
& -\frac{B \Delta_{l}^{B} h}{2 \rho Q^{3}},
\end{aligned}
$$$$
\frac{\partial^{2} C}{\partial B^{2}}=\frac{h}{Q \rho}+\frac{b}{Q \rho},
$$$$
\frac{\partial^{2} C}{\partial B \partial Q}=-\frac{B b}{\rho Q^{2}}-\frac{B h}{\rho Q^{2}}-\frac{\Delta_{h}^{B} b}{4 \rho Q^{2}}+\frac{\Delta_{l}^{B} b}{4 \rho Q^{2}}-\frac{\Delta_{h}^{B} h}{4 \rho Q^{2}}+\frac{\Delta_{l}^{B} h}{4 \rho Q^{2}} .
$$

Therefore we will obtain the following Hessian matrix

$$
\left.\begin{array}{c}
-\frac{B h}{\rho Q^{2}}-\frac{B h}{\rho Q^{2}}-\frac{\Delta_{h}^{B} b}{4 \rho Q^{2}}+\frac{\Delta_{l}^{B} b}{4 \rho Q^{2}}-\frac{\Delta_{h}^{B} h}{4 \rho Q^{2}}+\frac{\Delta_{l}^{B} h}{4 \rho Q^{2}} \\
\frac{2 K D}{Q^{3}}+\frac{K \Delta_{h}^{D}}{2 Q^{3}}-\frac{K \Delta_{l}^{D}}{2 Q^{3}}+\frac{B^{2} b}{\rho Q^{3}}+\frac{\Delta_{l}^{B} b}{6 \rho Q 3}+\frac{\Delta_{h}^{B^{2}} b}{6 \rho Q^{3}}+\frac{B^{2} h}{\rho Q^{3}} \\
+\frac{\Delta_{l}^{B^{2} h}}{6 \rho Q 3}+\frac{\Delta_{h}^{B^{2}} h}{6 \rho Q^{3}}+\frac{B \Delta_{h}^{B} b}{2 \rho Q^{3}}-\frac{B \Delta_{l}^{B} b}{2 \rho Q^{3}}+\frac{B \Delta_{h}^{B} h}{2 \rho Q^{3}}-\frac{B \Delta_{l}^{B} h}{2 \rho Q^{3}}
\end{array}\right]
$$

$$
\begin{aligned}
= & \frac{(h+b)^{2}}{\rho^{2} Q^{4}}\left(B^{2}+\frac{1}{6}\left(\Delta_{h}^{B^{2}}+\Delta_{l}^{B^{2}}\right)+\frac{B}{2}\left(\Delta_{h}^{B}-\Delta_{l}^{B}\right)\right) \\
& -\frac{(h+b)^{2}}{\rho^{2} Q^{4}}\left(B^{2}+\frac{1}{16}\left(\Delta_{h}^{B}-\Delta_{l}^{B}\right)^{2}+\frac{B}{2}\left(\Delta_{h}^{B}-\Delta_{l}^{B}\right)\right) \\
& +\frac{(h+b)}{\rho Q}\left(\frac{2 K D}{Q^{3}}+\frac{K \Delta_{h}^{D}}{2 Q^{3}}-\frac{K \Delta_{l}^{D}}{2 Q^{3}}\right),
\end{aligned}
$$

which can be simplified into

$$
\begin{aligned}
\operatorname{Det} 2= & \frac{(h+b)^{2}}{\rho^{2} Q^{4}}\left(\frac{5}{48} \Delta_{l}^{B^{2}}+\frac{5}{48} \Delta_{h}^{B^{2}}+\frac{1}{8} \Delta_{l}^{B} \Delta_{h}^{B}\right) \\
& +\frac{(h+b)}{\rho Q^{4}}\left(2 K D+\frac{1}{2} K \Delta_{h}^{D}-\frac{1}{2} K \Delta_{l}^{D}\right) .
\end{aligned}
$$


It is obvious that the first term is always greater than 0 . Now if the second term will be greater or equal to zero, we will have a strictly positive determinant. Thus

$$
\begin{aligned}
& \text { Det2 }>0 \text { if } \\
& 2 K D+\frac{1}{2} K \Delta_{h}^{D}-\frac{1}{2} K \Delta_{l}^{D} \geq 0 \Longleftrightarrow \Delta_{l}^{D} \leq \Delta_{h}^{D}+4 D .
\end{aligned}
$$

It is also obvious that the equation $\Delta_{l}^{D} \leq \Delta_{h}^{D}+4 D$ holds for every reasonable values of $\Delta_{l}^{D}$ in the calculations. If, on the contrary, $\Delta_{l}^{D}>\Delta_{h}^{D}+4 D$ there will be highly negative demands with a high degree of possibility. Therefore, to obtain the minimum of (16), (given the assumption in (21)) the system of two equations to be solved is given by

$$
\frac{\partial C^{*}}{\partial Q}=\frac{\partial C^{*}}{\partial B}=0 .
$$

First we look at the partial derivative in respect to $B$

$$
\begin{aligned}
& \frac{\partial C^{*}}{\partial B} \\
& =\frac{B b}{\rho Q}-b+\frac{B h}{\rho Q}+\frac{\Delta_{h}^{B} b}{4 \rho Q}-\frac{\Delta_{l}^{B} b}{4 \rho Q}+\frac{\Delta_{h}^{B} h}{4 \rho Q}-\frac{\Delta_{l}^{B} h}{4 \rho Q}=0 \\
& \Leftrightarrow B=\frac{\rho Q h-(1 / 4) \Delta_{h}^{B} b+(1 / 4) \Delta_{l}^{B} b-(1 / 4) \Delta_{h}^{B} h+(1 / 4) \Delta_{l}^{B} h}{h+b} \\
& =\frac{\rho Q h-(1 / 4)\left(\Delta_{h}^{B}-\Delta_{l}^{B}\right)(h+b)}{h+b} \\
& \Leftrightarrow B=\frac{\rho Q h}{h+b}-\frac{1}{4}\left(\Delta_{h}^{B}-\Delta_{l}^{B}\right) .
\end{aligned}
$$

Then we look at the partial derivative in respect to $Q$

$$
\begin{aligned}
\frac{\partial C^{*}}{\partial Q}= & -\frac{K D}{Q^{2}}-\frac{K \Delta_{h}^{D}}{4 Q^{2}}+\frac{K \Delta_{l}^{D}}{4 Q^{2}}-\frac{B^{2} b}{2 \rho Q^{2}}-\frac{\Delta_{l}^{B^{2}} b}{12 \rho Q^{2}}-\frac{\Delta_{h}^{B^{2}} b}{12 \rho Q^{2}} \\
& +\frac{\rho h}{2}-\frac{B^{2} h}{2 \rho Q^{2}}-\frac{\Delta_{l}^{B^{2}} h}{12 \rho Q^{2}}-\frac{\Delta_{h}^{B^{2} h}}{12 \rho Q^{2}} \\
& -\frac{B \Delta_{h}^{B} b}{4 \rho Q^{2}}+\frac{B \Delta_{l}^{B} b}{4 \rho Q^{2}}-\frac{B \Delta_{h}^{B} h}{4 \rho Q^{2}}+\frac{B \Delta_{l}^{B} h}{4 \rho Q^{2}}=0 \\
\Leftrightarrow \quad & -K \rho\left(D+\frac{1}{4} \Delta_{h}^{D}-\frac{1}{4} \Delta_{l}^{D}\right)-\frac{1}{2} B^{2}(h+b) \\
& -\frac{1}{12}\left(\Delta_{h}^{B^{2}}-\Delta_{l}^{B^{2}}\right)(h+b) \\
& -\frac{1}{4} B\left(\Delta_{h}^{B}-\Delta_{l}^{B}\right)(h+b)+\frac{h \rho^{2} Q^{2}}{2}=0
\end{aligned}
$$

Substituting $B$ in (24) given by (23) will give us

$$
\begin{aligned}
&- K \rho\left(D+\frac{1}{4} \Delta_{h}^{D}-\frac{1}{4} \Delta_{l}^{D}\right) \\
&- \frac{1}{2}\left(\frac{Q^{2} h^{2}}{(h+b)^{2}}-\frac{Q h\left(\Delta_{h}^{B}-\Delta_{l}^{B}\right)}{2(h+b)}+\frac{\left(\Delta_{h}^{B}-\Delta_{l}^{B}\right)^{2}}{16}\right)(h+b) \\
&- \frac{1}{12}\left(\Delta_{h}^{B^{2}}+\Delta_{l}^{B^{2}}\right)(h+b) \\
&- \frac{1}{4}\left(\frac{Q h}{h+b}-\frac{1}{4}\left(\Delta_{h}^{B}-\Delta_{l}^{B}\right)\right)\left(\Delta_{h}^{B}-\Delta_{l}^{B}\right)(h+b) \\
&+ \frac{h \rho^{2} Q^{2}}{2}=0 \\
& \Longleftrightarrow-K \rho\left(D+\frac{1}{4} \Delta_{h}^{D}-\frac{1}{4} \Delta_{l}^{D}\right)-\frac{\rho^{2} Q^{2} h^{2}}{2(h+b)}+\frac{Q h\left(\Delta_{h}^{B}-\Delta_{l}^{B}\right)}{4} \\
&-\frac{\left(\Delta_{h}^{B}-\Delta_{l}^{B}\right)^{2}(h+b)}{32}-\frac{1}{12}\left(\Delta_{h}^{B^{2}}+\Delta_{l}^{B^{2}}\right)(h+b) \\
&+\frac{Q \rho h\left(\Delta_{h}^{B}-\Delta_{l}^{B}\right)}{4}+\frac{1}{16}\left(\Delta_{h}^{B}-\Delta_{l}^{B}\right)^{2}(h+b) \\
&+\frac{h \rho^{2} Q^{2}}{2}=0 \\
& \Leftrightarrow Q^{2}\left(\frac{h p \rho^{2}}{2(h+b)}\right) \\
&(\left.\frac{\left(\Delta_{h}^{B} h\right.}{2}-\frac{\rho^{2} h}{2(h+b)}\right)=K \rho(D+b) \\
& \rho_{l}^{2} h^{2} \\
& 32
\end{aligned}
$$

It is worth noticing that $Q^{2}>0$ under the conditions given by the definition. Equation (25) collapses into

$$
Q_{f}^{*}=\sqrt{\frac{2 K D}{\rho b}+\frac{2 K D}{\rho h}+\frac{K \Delta_{h}^{D}}{2 \rho b}+\frac{K \Delta_{h}^{D}}{2 \rho h}-\frac{K \Delta_{l}^{D}}{2 \rho b}-\frac{K \Delta_{l}^{D}}{2 \rho h}+\frac{5 \Delta_{h}^{B^{2}} h}{48 \rho^{2} b}+\frac{5 \Delta_{h}^{B^{2}}}{24 \rho^{2}}+\frac{5 \Delta_{h}^{B^{2}} b}{48 \rho^{2} h}+\frac{5 \Delta_{l}^{B^{2}} h}{48 \rho^{2} b}+\frac{5 \Delta_{l}^{B^{2}}}{24 \rho^{2}}+\frac{5 \Delta_{l}^{B^{2}} b}{48 \rho^{2} h}+\frac{6 \Delta_{h}^{B} \Delta_{l}^{B} h}{48 \rho^{2} b}+\frac{6 \Delta_{h}^{B} \Delta_{l}^{B}}{24 \rho^{2}}+\frac{6 \Delta_{h}^{B} \Delta_{l}^{B} b}{48 \rho^{2} h}} .
$$


Finally (26) can be rewritten into

$$
\begin{aligned}
& Q_{f}^{*} \\
& =\sqrt{\frac{2 K D}{h \rho} \cdot \frac{h+b}{b}+\frac{K}{2 \rho h} \cdot \frac{h+b}{b} \cdot\left(\Delta_{h}^{D}-\Delta_{l}^{D}\right)+\frac{h+b}{48 \rho^{2} h} \cdot \frac{h+b}{b} \cdot\left(5 \Delta_{h}^{B^{2}}+5 \Delta_{l}^{B}+6 \Delta_{h}^{B} \Delta_{l}^{B}\right)} .
\end{aligned}
$$

It is worth noticing that terms $3-13$ in (26) or the second two terms in (27) all origin from the uncertainties in the demand and the setup times. It is also worth noticing that the fuzzy optimal order quantity always increases with the uncertaintiesin the setup times, whereas the uncertainties in the demand may have a different impact on the order quantity. This is similar to the results found by Björk [16]. Let us investigate what happens with (26) and (27) given a zero distribution in the fuzzy numbers (i.e., all fuzzy numbers are crisp). Equation (26) will then collapse into

$$
\begin{aligned}
Q_{c}^{*} & =\sqrt{\frac{2 K D}{\rho b}+\frac{2 K D}{\rho h}}=\sqrt{\frac{2 b K D+2 h K D}{b h \rho}} \\
& =\sqrt{\frac{2 K D}{h \rho}} \cdot \sqrt{\frac{h+b}{b}} .
\end{aligned}
$$

This can also be seen directly from (27). It is worth noticing that (28) is identical to the crisp solution given in the basic EOQ literature (Cárdenas-Barrón, [22] for instance). From (23) we also find that

$$
\begin{aligned}
B_{f}^{*} & \\
& =\frac{\rho h}{h+b} \cdot Q_{F}^{*}-\frac{1}{4}\left(\Delta_{h}^{B}-\Delta_{l}^{B}\right),
\end{aligned}
$$

which will ultimately give

$$
B_{f}^{*}=\frac{\rho h}{h+b} \sqrt{\frac{2 K D}{h \rho} \cdot \frac{h+b}{b}+\frac{K}{2 \rho h} \cdot \frac{h+b}{b} \cdot\left(\Delta_{h}^{D}-\Delta_{l}^{D}\right)+\frac{h+b}{48 \rho^{2} h} \cdot \frac{h+b}{b} \cdot\left(5 \Delta_{h}^{B^{2}}+5 \Delta_{l}^{B^{2}}+6 \Delta_{h}^{B} \Delta_{l}^{B}\right)}-\frac{1}{4}\left(\Delta_{h}^{B}-\Delta_{l}^{B}\right) .
$$

In (30), we can also find that if all uncertainties are 0 , then the optimal $B$ will be

$$
B_{c}^{*}=\frac{\rho h}{h+b} \sqrt{\frac{2 K D}{\rho h} \cdot \frac{h+b}{b}}=\sqrt{\frac{2 K D \rho h}{b(h+b)}},
$$

which is the crisp solution according to basic EOQ literature.

\section{Numerical Example}

Let us assume that we have a paper producer that wants to determine how much should be produced of a certain product (at each production run). The total annual demand is assumed to be at $800000 \mathrm{~kg}(D)$ with a $\Delta_{l}^{D}$ of $20000 \mathrm{~kg}$ and a $\Delta_{h}^{D}$ of $40000 \mathrm{~kg}$. The total production capacity per year is $2000000 \mathrm{~kg}(P)$. The paper cost is 1 euro $/ \mathrm{kg}$. There is a fixed cost at each production setup: 2000 euro. The holding costs are $25 \%$ of the purchase price, that is, 0.25 euro per $\mathrm{kg}$ and annum $(h)$. The penalty costs are 5 euro per $\mathrm{kg}$ and annum (b). This penalty cost represents a service level about 95\% (if a simple normal distributed stochastic demand is assumed), but as stated earlier, we assume fuzzy demand in this model and the service level equivalence is given as a comparison only. The uncertainty in the setup times (from the setups and also from the uncertain raw material availability) is typically two days in the lower direction and 5 days in the upper direction. Therefore, $\Delta_{l}^{B}$ is $4383.56 \mathrm{~kg}$ (or 2 days demand) and a $\Delta_{h}^{B}$ of $10958.90 \mathrm{~kg}$ (or 5 days demand). Given these parameters, the optimization results for this example are given in Table 1.

Note that the results in Table 1 indicate that the order size would increase with $3.1 \%$ if the uncertainties are accounted for in appropriate manner. This would require an increased total cost of $3.1 \%$, when the backorder level is decreased $35 \%$. The decrease in the backorder level comes from the asymmetric triangular fuzzy $\Delta^{B}$ and $\Delta^{D}$. Given these parameters, the solution does not seem very sensitive to the batch size or backorder level, which is also quite expected. In addition, if the crisp solution for the batch size and backorder level is used instead of the fuzzy optimal solutions, the fuzzy objective value would only increase from 22037.37 to 22126.44 . This is only an increase in $0.4 \%$. These figures also support the claim that the solution is not so sensitive (in the parameter settings used in the example). However, the model is generic and can be used in many different situations 
TABLE 1: The result from the example calculations.

\begin{tabular}{lccc}
\hline & $Q^{*}$ & $B^{*}$ & $C^{*}$ \\
\hline Crisp & 149666.30 & 4276.18 & 21380.90 \\
Fuzzy & 154261.62 & 2763.64 & 22037.37 \\
\hline change & $3.07 \%$ & $-35.37 \%$ & $3.07 \%$ \\
\hline
\end{tabular}

in other industrial environments. For these settings, the impact of fuzzy setup times may be more significant. In the following, a short sensitivity analysis is conducted. Each of the $\Delta$ 's is changed (either halved or doubled) and the changes (in percentage) from the original fuzzy solution values are calculated. From Table 2, it can be seen that the change in the solution is very marginal if the $\Delta^{D}$ parameters are changed. This is natural from the definition of the fuzzy demand in the model: for instance, symmetrical triangular fuzzy demand will not have any impact on the solution; that is, the upper distribution will eliminate the effect of the lower distribution. The changes in the $\Delta^{B}$ parameters will, however, have a greater impact on the fuzzy solution values. Therefore it may be more important for managers to focus on reducing the uncertainties in the setup times than the uncertainties in the demand.

\section{Discussion and Further Research}

Making the right decision in a production-distribution network may be the key to success. There have been many contributions regarding the replenishment decisions under the continuous-review policy. Still it is necessary to make certain improvement to this track of research in order to meet the inherent fuzzy uncertainties in the applications. This paper contributes to the theory in this field by presenting the analytical solution for a case, where backorders are allowed, the production rate is finite, and the production setup times as well as the demand rates are inherent fuzzy numbers. The fuzzy numbers are allowed to be asymmetric, which complicates the analytical solution compared to the solution of the model found in Björk [18]. The results from the research consist of the proof of convexity and the analytical solution after the fuzzy model has been defuzzified with the signed difference method. The analytical solution is coherent with the previous findings within the EOQ literature; for instance, the fuzzy solution collapses to the crisp solution, if the uncertainties are assumed to be zero. The model is also tested with a theoretical case example from a paper-producer. The process industry applications have served as a source of inspiration in solving the fuzzy EPQ problems, even if the results in this paper are theoretical. The model is concluded with a brief sensitivity analysis of the model, where it was concluded that it is likely that it would be more crucial to reduce the uncertainty in the setup times than the uncertainty in the demand (for the parameter settings given in the example).

The model could, in the future, be extended to cover more membership functions than the triangular one. In addition, different case studies that give rise to a systematic
TABLE 2: The results from the sensitivity analysis, that is, the changes in the fuzzy solution values, given some changes in the fuzzy parameters $\Delta^{B}$ and $\Delta^{D}$.

\begin{tabular}{lccc}
\hline Parameter change & $Q^{*}$ & $B^{*}$ & $C^{*}$ \\
\hline $2 \Delta_{L}^{D}$ & $-0.29 \%$ & $-0.47 \%$ & $-0.29 \%$ \\
$0.5 \Delta_{L}^{D}$ & $0.15 \%$ & $0.23 \%$ & $0.15 \%$ \\
\hline $2 \Delta_{h}^{D}$ & $0.59 \%$ & $0.94 \%$ & $0.59 \%$ \\
$0.5 \Delta_{h}^{D}$ & $-0.29 \%$ & $-0.47 \%$ & $-0.29 \%$ \\
\hline $2 \Delta_{L}^{B}$ & $1.53 \%$ & $42.10 \%$ & $1.53 \%$ \\
$0.5 \Delta_{L}^{B}$ & $-0.58 \%$ & $-20.75 \%$ & $-0.58 \%$ \\
\hline $2 \Delta_{h}^{B}$ & $5.45 \%$ & $-90.44 \%$ & $5.45 \%$ \\
$0.5 \Delta_{h}^{B}$ & $-1.61 \%$ & $47.00 \%$ & $-1.61 \%$ \\
\hline
\end{tabular}

way of obtaining the membership functions could be considered. Finally, the safety stock should be quantified under the fuzzy uncertainties in order to obtain a complete inventory decision model for the proposed problem.

\section{Appendix}

In this appendix, the basics of fuzzy numbers as well as the signed distance method are given in order to make the modeling effort self-contained.

Definition 1. Consider the fuzzy set $\tilde{A}=(a, b, c)$ where $a<$ $b<c$ and defined on $R$, which is called a triangular fuzzy number, if the membership function of $\widetilde{A}$ is given by

$$
\mu_{\widetilde{A}}(x)= \begin{cases}\frac{(x-a)}{(b-a)}, & a \leq x \leq b \\ \frac{(c-x)}{(c-b)}, & b \leq x \leq c \\ 0, & \text { otherwise. }\end{cases}
$$

Definition 2. Let $\widetilde{B}$ be a fuzzy set on $R$ and $0 \leq \alpha \leq 1$. The $\alpha$-cut of $\widetilde{B}$ is all the points $x$ such that $\mu_{\widetilde{B}}(x) \geq \alpha$, that is,

$$
B(\alpha)=\left\{x \mid \mu_{\widetilde{B}}(x) \geq \alpha\right\} .
$$

In order to find non-fuzzy values for the model in the next section we need to use some distance measures, and as in Chang [9] we will use the signed distance (Yao and $\mathrm{Wu}[26]$ ).

Definition 3. For any $a$ and $0 \in R$, the signed distance from $a$ to 0 is $d_{0}(a, 0)=a$. And if $a<0$, the distance from $a$ to 0 is $-a=-d_{0}(a, 0)$.

Let $\Omega$ be the family of all fuzzy sets $\widetilde{B}$ defined on $R$ for which the $\alpha$-cut $B(\alpha)=\left[B_{L}(\alpha), B_{U}(\alpha)\right]$ exists for every $\alpha \in$ $[0,1]$, and both $B_{L}(\alpha)$ and $B_{U}(\alpha)$ are continuous functions on $\alpha \in[0,1]$. Then, for any $\widetilde{B} \in \Omega$, we have (see Chang, [9])

$$
\widetilde{B}=\bigcup_{0 \leq \alpha \leq 1}\left[B_{L}(\alpha)_{\alpha}, B_{U}(\alpha)_{\alpha}\right] .
$$

From Chang [9], it can be finally stated (originally by results from Yao and $\mathrm{Wu}[26]$ ) how to calculate the signed distances. 
Definition 4. For $\widetilde{B} \in \Omega$ define the signed distance of $\widetilde{B}$ to $\widetilde{0}_{1}$ as

$$
d\left(\widetilde{B}, \tilde{0}_{1}\right)=\frac{1}{2} \int_{0}^{1}\left[B_{L}(\alpha)+B_{U}(\alpha)\right] d \alpha
$$

\section{References}

[1] F. W. Harris, "How many parts to make at once," The Magazine of Management, vol. 10, pp. 135-136, 1913.

[2] M. J. Liberatore, "The EOQ model under stochastic lead time," Operations Research, vol. 27, pp. 391-396, 1979.

[3] A. L. Guiffrida, "Fuzzy inventory models," in Inventory Management: Non-Classical Views, M. Y. Jaber, Ed., chapter 8, pp. 173-198, CRC Press, Boca Raton, Fla, USA, 2009.

[4] L. A. Zadeh, "Fuzzy sets," Information and Control, vol. 8, no. 3, pp. 338-353, 1965.

[5] L. A. Zadeh, "Outline of a new approach to the analysis of complex systems and decision processes," IEEE Transactions on Systems, Man and Cybernetics, vol. 3, no. 1, pp. 28-44, 1973.

[6] L. Y. Ouyang and K. S. Wu, "A minimax distribution free procedure for mixed inventory model with variable lead time," International Journal of Production Economics, vol. 56-57, pp. 511-516, 1998.

[7] L. Y. Ouyang and J. S. Yao, "A minimax distribution free procedure for mixed inventory model involving variable lead time with fuzzy demand," Computers and Operations Research, vol. 29, no. 5, pp. 471-487, 2001.

[8] M. K. Salameh and M. Y. Jaber, "Economic production quantity model for items with imperfect quality," International Journal of Production Economics, vol. 64, no. 1, pp. 59-64, 2000.

[9] H. C. Chang, "An application of fuzzy sets theory to the EOQ model with imperfect quality items," Computers and Operations Research, vol. 31, no. 12, pp. 2079-2092, 2004.

[10] M. Khan, M. Y. Jaber, A. L. Guiffrida, and S. Zolfaghari, "A review of the extensions of a modified EOQ model for imperfect quality items," International Journal of Production Economics, vol. 132, no. 1, pp. 1-12, 2011.

[11] M. Y. Jaber, M. Bonney, and I. Moualek, "An economic order quantity model for an imperfect production process with entropy cost," International Journal of Production Economics, vol. 118, no. 1, pp. 26-33, 2009.

[12] M. Khan, M. Y. Jaber, and M. I. M. Wahab, "Economic order quantity model for items with imperfect quality with learning in inspection," International Journal of Production Economics, vol. 124, no. 1, pp. 87-96, 2010.

[13] M. Khan, M. Y. Jaber, and M. Bonney, "An economic order quantity (EOQ) for items with imperfect quality and inspection errors," International Journal of Production Economics, vol. 199, no. 1, pp. 113-118, 2010.

[14] C. Carlsson and R. Fuller, "Soft computing and the Bullwhip effect," Economics and Complexity, vol. 2, no. 3, pp. 1-26, 1999.

[15] K. M. Björk and C. Carlsson, "The outcome of imprecise lead times on the distributors," in Proceedings of the 38th Annual Hawaii International Conference on System Sciences (HICSS '05), pp. 81-90, 2005, Track 3.

[16] K. M. Björk, "An analytical solution to a fuzzy economic order quantity problem," International Journal of Approximate Reasoning, vol. 50, no. 3, pp. 485-493, 2009.

[17] S. C. Chang, J. S. Yao, and H. M. Lee, "Economic reorder point for fuzzy backorder quantity," European Journal of Operational Research, vol. 109, pp. 183-202, 1998.
[18] K.-M. Björk, "A fuzzy economic production quantity problem with back orders," in Proceedings of the World Conference on Soft Computing (WConSC '11), 2011.

[19] J. S. Yao, L. Y. Ouyang, and H. C. Chang, "Models for a fuzzy inventory of two replaceable merchandises without backorder based on the signed distance of fuzzy sets," European Journal of Operational Research, vol. 150, no. 3, pp. 601-616, 2003.

[20] J. S. Yao and J. Chiang, "Inventory without backorder with fuzzy total cost and fuzzy storing cost defuzzified by centroid and signed distance," European Journal of Operational Research, vol. 148, no. 2, pp. 401-409, 2003.

[21] K.-M. Björk, "A multi-item fuzzy economic production quantity problem with a finite production rate," International Journal of Production Economics, vol. 135, no. 2, pp. 702-707, 2012.

[22] L. E. Cárdenas-Barrón, "The economic production quantity (EPQ) with shortage derived algebraically," International Journal of Production Economics, vol. 70, no. 3, pp. 289-292, 2001.

[23] L. E. Cárdenas-Barrón, "An easy method to derive EOQ and EPQ inventory models with backorders," Computers and Mathematics with Applications, vol. 59, no. 2, pp. 948-952, 2010.

[24] L. E. Cárdenas-Barrón, “The derivation of EOQ/EPQ inventory models with two backorders costs using analytic geometry and algebra," Applied Mathematical Modelling, vol. 35, no. 5, pp. 2394-2407, 2011.

[25] N. Kazemi, E. Ehsani, and M. Y. Jaber, "An inventory model with backorders with fuzzy parameters and decision variables," International Journal of Approximate Reasoning, vol. 51, no. 8, pp. 964-972, 2010.

[26] J. S. Yao and K. Wu, "Ranking fuzzy numbers based on decomposition principle and signed distance," Fuzzy Sets and Systems, vol. 116, pp. 275-288, 2000. 

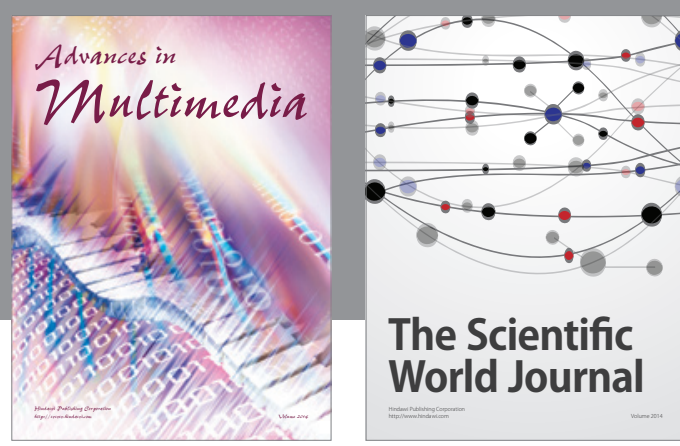

The Scientific World Journal
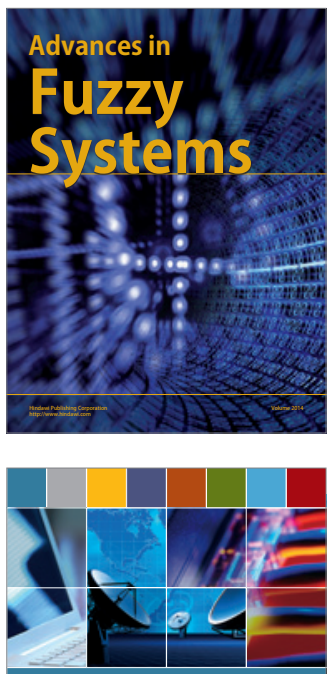

Computer Networks and Communications
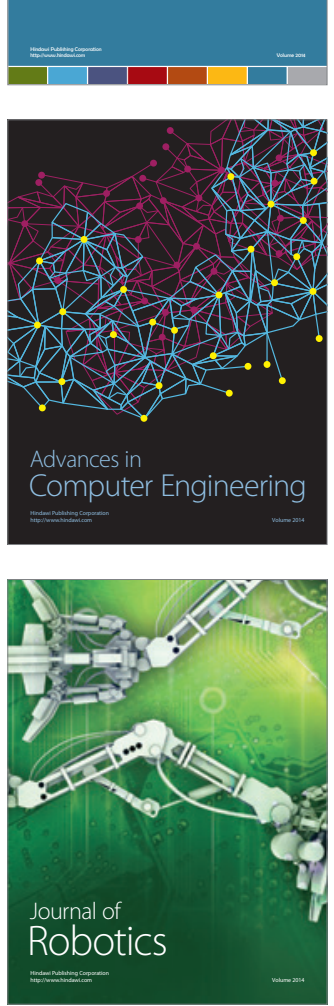
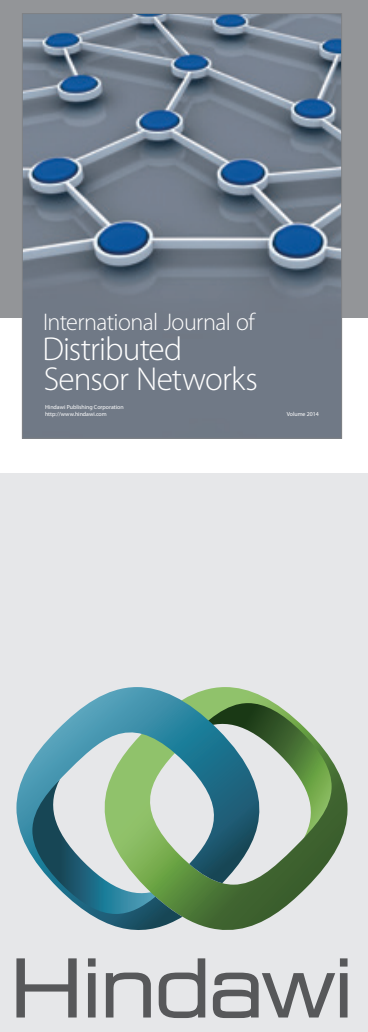

Submit your manuscripts at

http://www.hindawi.com
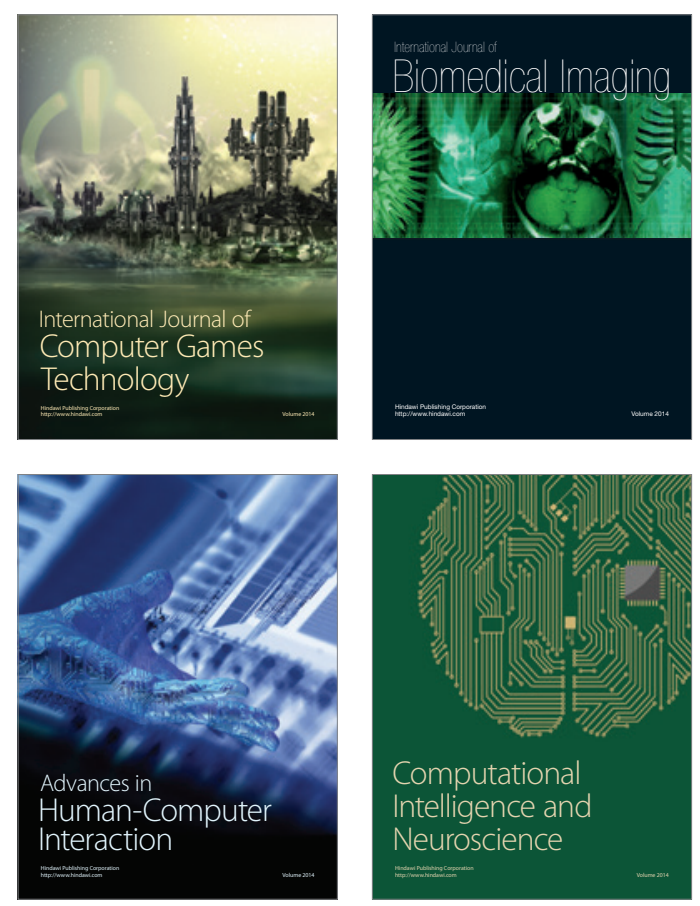
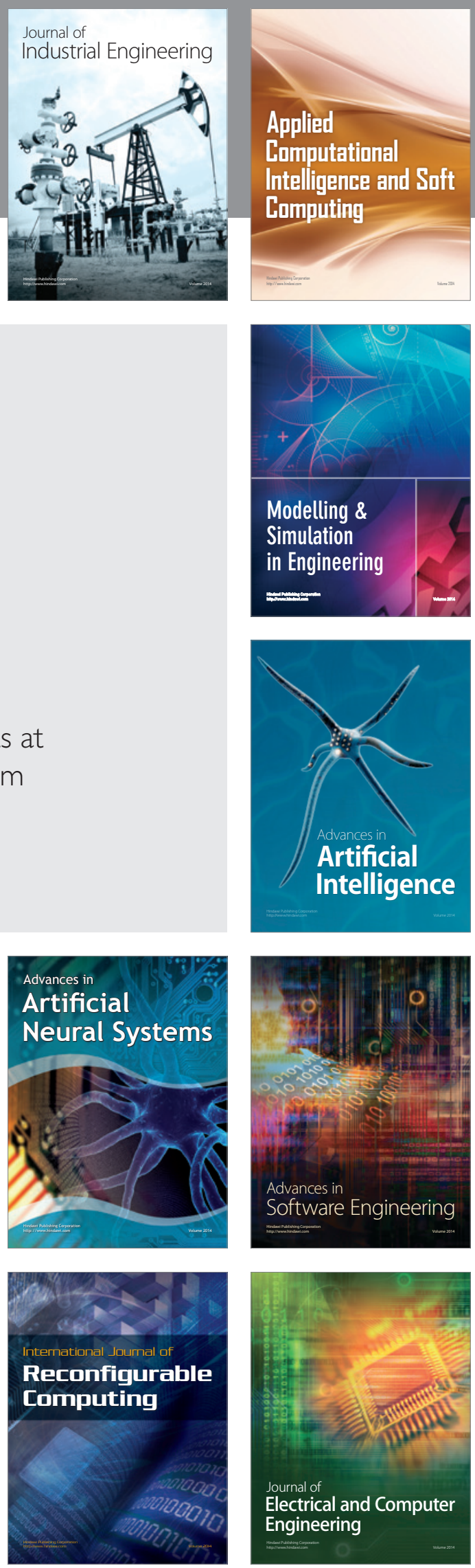